\title{
Electron Probe Microanalysis of Exogenous Pigmentation of Oral Mucosa Originating from Dental Alloy: Two Case Reports
}

\author{
Akinori Funayama1 ${ }^{*}$, Toshihiko Mikami' ${ }^{1}$ Kanae Niimi' ${ }^{1}$, Hiroyuki Kano ${ }^{1,2}$, Yutaka Nikkuni ${ }^{3}$ \\ Manabu Yamazaki ${ }^{4}$, Tadaharu Kobayashi ${ }^{1}$ \\ ${ }^{1}$ Division of Reconstructive Surgery for Oral and Maxillofacial Region, Niigata University Graduate School of \\ Medical and Dental Sciences, Niigata, Japan \\ ${ }^{2}$ Division of Oral and Maxillofacial Surgery, Uonuma Kikan Hospital, Minamiuonuma City, Japan \\ ${ }^{3}$ Division of Oral and Maxillofacial Radiology, Niigata University Graduate School of Medical and Dental \\ Sciences, Niigata, Japan \\ ${ }^{4}$ Division of Oral Pathology, Niigata University Graduate School of Medical and Dental Sciences, Niigata, Japan \\ Email: "funayama@dent.niigata-u.ac.jp
}

Received 25 March 2016; accepted 24 April 2016; published 27 April 2016

Copyright (C) 2016 by authors and Scientific Research Publishing Inc.

This work is licensed under the Creative Commons Attribution International License (CC BY). http://creativecommons.org/licenses/by/4.0/

c) (i) Open Access

\begin{abstract}
Pigmentation of the oral mucosa is relatively common and has a wide variety of etiologies. Although most pigmented lesions of the oral mucosa are associated with deposition of melanin and accidental displacement of a dental alloy, accurate differential diagnosis of a pigmented lesion is important, especially in the case of malignant melanoma. We report two cases of oral mucosal pigmentation associated with accidental displacement of a dental alloy in which malignant melanoma was suspected. Excisional biopsy was carried out in these cases with the incision line set at approximately $5 \mathrm{~mm}$ from the lesions. Histopathologically, brownish foreign substances were observed in the lamina propria. Metal quantitative analyses of the extracted specimens were carried out by electron probe microanalysis (EPMA). The metal components and the mass concentration revealed that the metals were derived from a dental casting silver alloy in Case 1 and from a gold-silver palladium alloy in Case 2. Although exogenous pigmentation originating from a dental alloy is not rare, differential diagnosis of oral pigmented lesions is sometimes very difficult. In such cases, histopathological examination may be necessary for the diagnosis to exclude melanocytic lesions and EPMA may be effective to identify the causative dental alloy.
\end{abstract}

\section{Keywords}

Intraoral Pigmentation, Oral Melanoma, Dental Alloy, Oral Mucosa

\footnotetext{
${ }^{*}$ Corresponding author.
}

How to cite this paper: Funayama, A., Mikami, T., Niimi, K., Kano, H., Nikkuni, Y., Yamazaki, M. and Kobayashi, T. (2016) Electron Probe Microanalysis of Exogenous Pigmentation of Oral Mucosa Originating from Dental Alloy: Two Case Reports. Open Journal of Stomatology, 6, 120-126. http://dx.doi.org/10.4236/ojst.2016.64015 


\section{Introduction}

Pigmentation of the oral mucosa is distinguished as either endogenous or exogenous. The causes of each are endogenous pigments of melanin, hemosiderin, or bilirubin and exogenous pigments of metal or medical agents [1]. Although most pigmented lesions of the oral mucosa are associated with deposition of melanin and accidental displacement of a dental alloy, accurate differential diagnosis of a pigmented lesion is important. It is particularly important to differentiate between pigmented nevus caused by the proliferation of nevus cells and malignant melanoma derived from melanocytes. Therefore, histopathological examinations are required [1]-[3].

We report two cases of oral mucosal pigmentation associated with accidental displacement of a dental alloy in which malignant melanoma was suspected and excisional biopsy and electron probe microanalysis were carried out.

\section{Case Report}

\subsection{Case 1}

A 65-year-old female visited our hospital with the complaint of a dark lesion in the right retromolar region. The patient's medical history was myasthenia gravis, thyroid gland tumor, cervical spinal canal stenosis, and primary obstructive cirrhosis. About two years prior to visiting our hospital, the patient had damaged the patient's distal gingiva of the lower wisdom tooth by a diamond bar upon undergoing a crown preparation of the wisdom tooth that was mounted by silver alloy construction.

Physical examination revealed that the patient had symmetric facial appearance and no abnormal findings in cervical lymph nodes. Three dark lesions were observed in the oral mucosa around the right lower wisdom tooth (Figure 1). A well-defined, dark black, elastic softening, bulging lesion, $12 \times 8 \mathrm{~mm}$ in size, was observed from the distal side of the wisdom tooth towards the inner side, with a small piece of palpable bone-like hard tissue inside (Figure 1(a)). An ill-defined, flat, dark lesion, $5 \times 4 \mathrm{~mm}$ in size, was observed on the buccal mucosa of the distal outer side of the wisdom tooth (Figure 1(b)), along with a slightly dark lesion of $2 \times 2 \mathrm{~mm}$ in size, on the buccal gingiva (Figure 1(c)).

Widening of the periodontal ligament space of the right wisdom tooth and radio-opacity at the anterior margin of the mandibular ramus was observed by dental X-ray imaging, though bone resorption was not observed at the distal site of the wisdom tooth (Figure 2(a)). Although an enhanced lesion and bone resorption were not observed by contrast-enhanced CT, a small piece with a similar level of opacity as that of the cortical bone was observed on the anteromedial side of the right mandibular ramus (Figure 2(b)).

There was clinical suspicion of exogenous pigmentation, pigmented nevus, or malignant melanoma. Therefore, excisional biopsy for differential diagnosis was carried out with extraction of the right lower wisdom tooth and integral excision of the three lesions including the periosteum with a safety margin of approximately $5 \mathrm{~mm}$ from the dark lesions (Figure 3). Upon confirmation of no dark lesions on the resection stumps, primary suturing of the wound was carried out.

Exogenous pigmentation was diagnosed on the basis of histopathological findings of brownish foreign substances in the lamina propria that were granular in shape and light brown at the outer periphery (Figure 4). Fibrosis was also observed in the lamina propria.

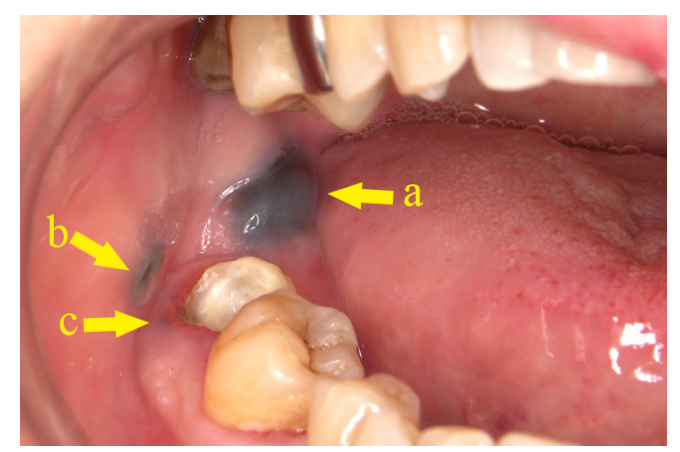

Figure 1. Preoperative intraoral view in Case 1: Three dark lesions were observed in the oral mucosa around her right mandiblar wisdom tooth. 

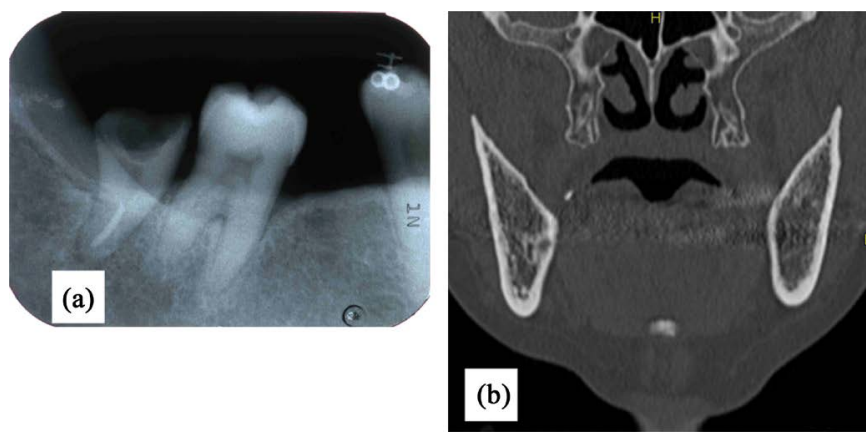

Figure 2. Preoperative image findings in Case 1: (a) Radio-opacity was observed at the anterior margin of the mandibular ramus by dental X-ray imaging and (b) a small piece with a level of opacity similar to that of the cortical bone was observed on the anteromedial side of the right mandibular ramus.
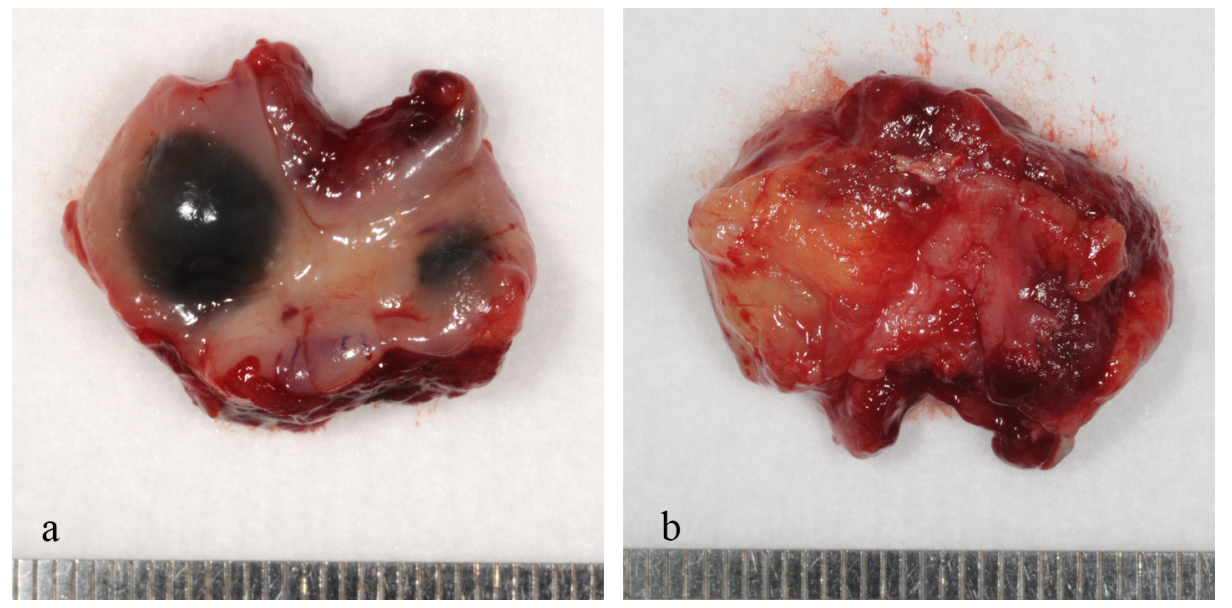

Figure 3. Resected specimen in Case 1: (a) Excisional biopsy of three lesions as a lump was carried out with the incision line set at approximately $5 \mathrm{~mm}$ outside the dark lesions and (b) no dark lesions were observed on the stump.
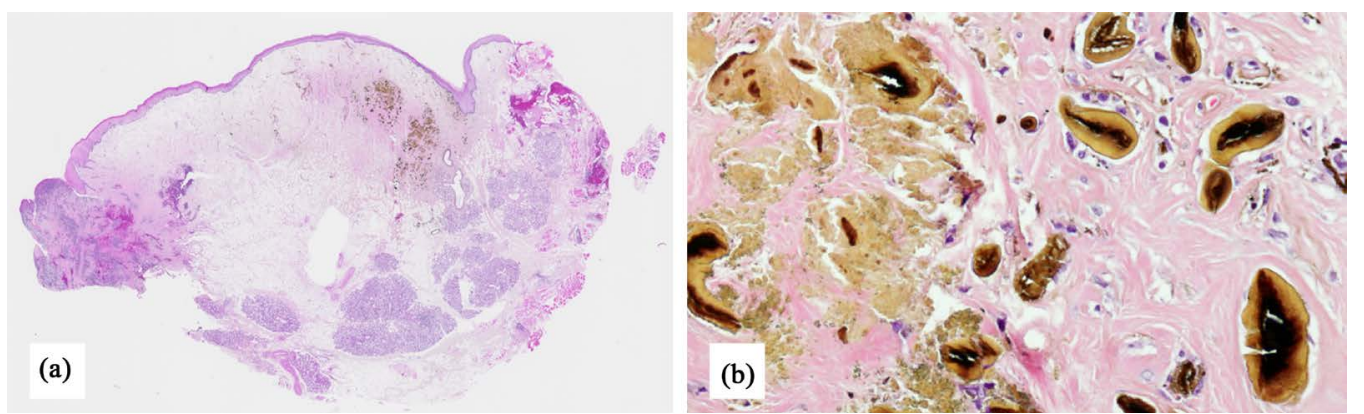

Figure 4. Histopathological images (H \& E stain) in Case 1: Brownish foreign substances and fibrosis were in the lamina propria (original magnification $(\mathrm{a}): \times 2,(\mathrm{~b}): \times 100)$

Electron probe microanalysis (EPMA) was performed using an EMPA-8705 (Shimadzu Corporation). The metal components were silver (69.2\%), indium (6.4\%), tin (11.3\%), and zinc (13.1\%) (Figure 5). The results showed that the metal was a dental casting silver alloy used for abutment construction.

One month post-operatively, the area had healed normally and the last follow-up with the patient was 3 months after the surgery uneventfully.

\subsection{Case 2}

A 45-year-old female visited our hospital with the complaint of a dark lesion on the right floor of the mouth. The 
patiant's medical history was duodenal ulcer. About six years ago, she visited our hospital for a detailed examination of a hemangioma in the left buccal mucosa. Because there were no symptoms, the lesion was followed up without treatment by her local dentist. Recently, the patient became aware of a dark lesion on the right floor of the mouth and consulted her regular dentist, who recommended a detailed examination.

Physical examination revealed that the patient had symmetric facial appearance and no abnormal findings in cervical lymph nodes. A well-defined dark lesion, with a diameter of approximately $5 \mathrm{~mm}$, was observed in the right floor of the mouth, and the lesion was slightly bulging but with no induration (Figure 6). No opacity was observed in the floor of the mouth by panoramic X-ray and CT images (Figure 7).

There was clinical suspicion of exogenous pigmentation, pigmented nevus, or malignant melanoma. Therefore, excisional biopsy for differential diagnosis was carried out under general anesthesia with extraction of the hemangioma of the left buccal mucosa. The pigmented lesion was excised while preserving the sublingual gland with a safety margin of approximately $5 \mathrm{~mm}$ from the dark area on the right floor of the mouth (Figure 8(a)). While a dark color was observed on the bottom of resection stump (Figure 8(b)), no dark lesion was detected on the exposed sublingual gland, and primary suturing of the wound was carried out.

Histopathologically, there was slight bulging of the mucosa along with formation of lymphoid tissues underneath the mucosal epithelium, in which blackish-brown foreign substances were sporadically observed as well as pigmented dark granules in the collagen fibril (Figure 9). The blackish-brown foreign substances were ellip-
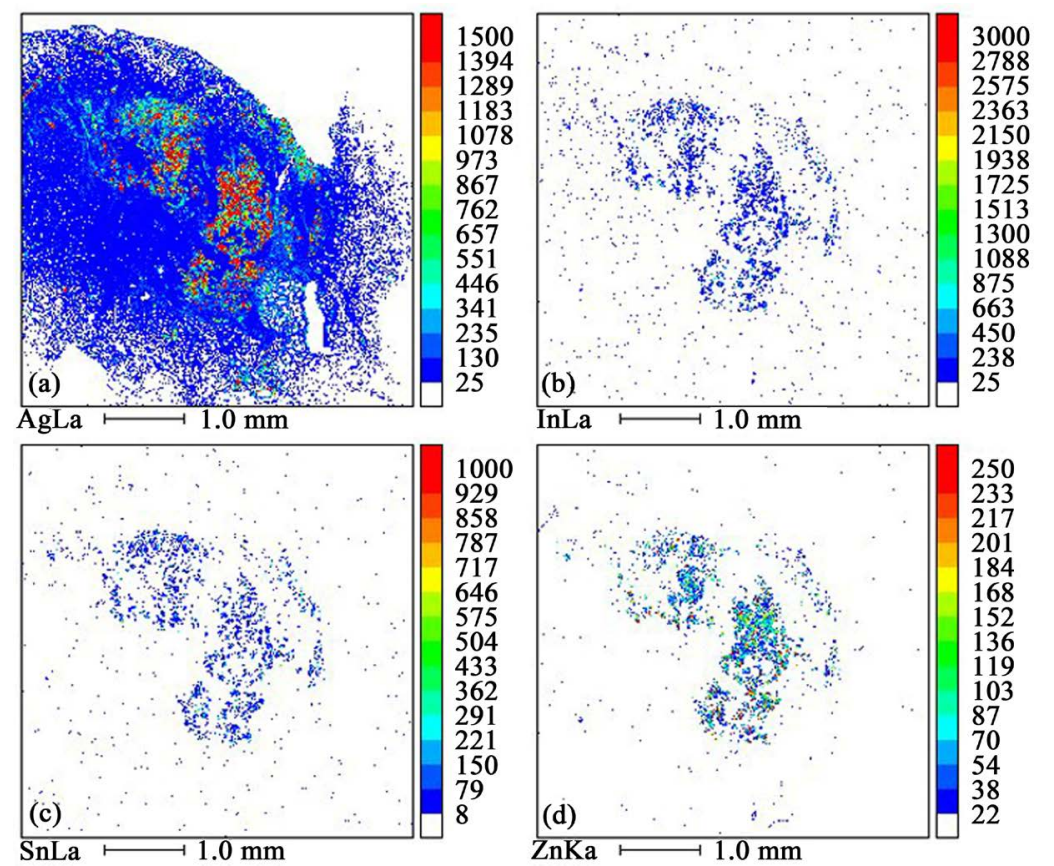

Figure 5. Electron probe microanalysis (EPMA) in Case 1: (a) The metal components were silver 69.2\%; (b) indium (6.4\%); (c) tin (11.3\%); and (d) zinc (13.1\%).

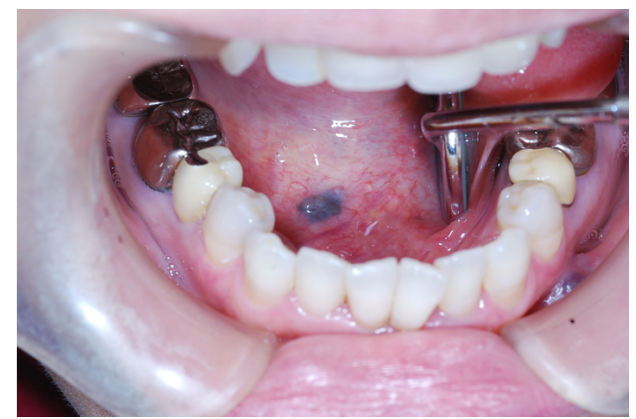

Figure 6. Preoperative intraoral view in Case 2: A well-defined dark lesion was observed in the right floor of the mouth. 


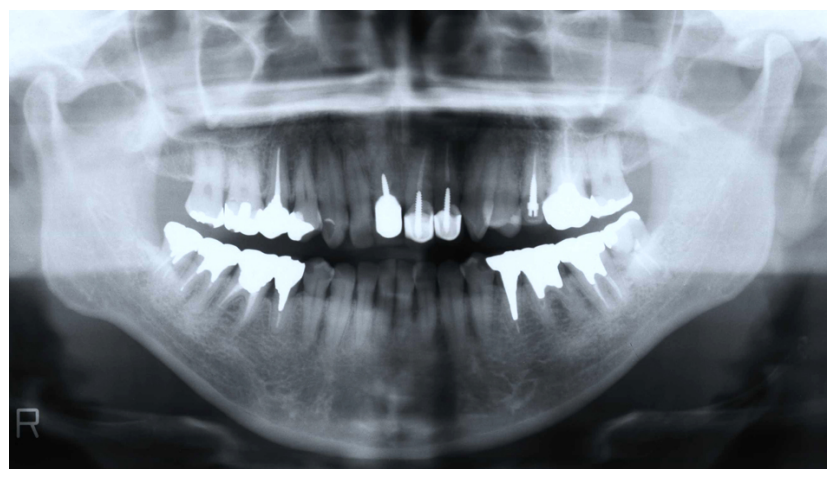

Figure 7. Preoperative image findings in Case 2: No opacity was observed in the floor of the mouth by panoramic X-ray imaging.
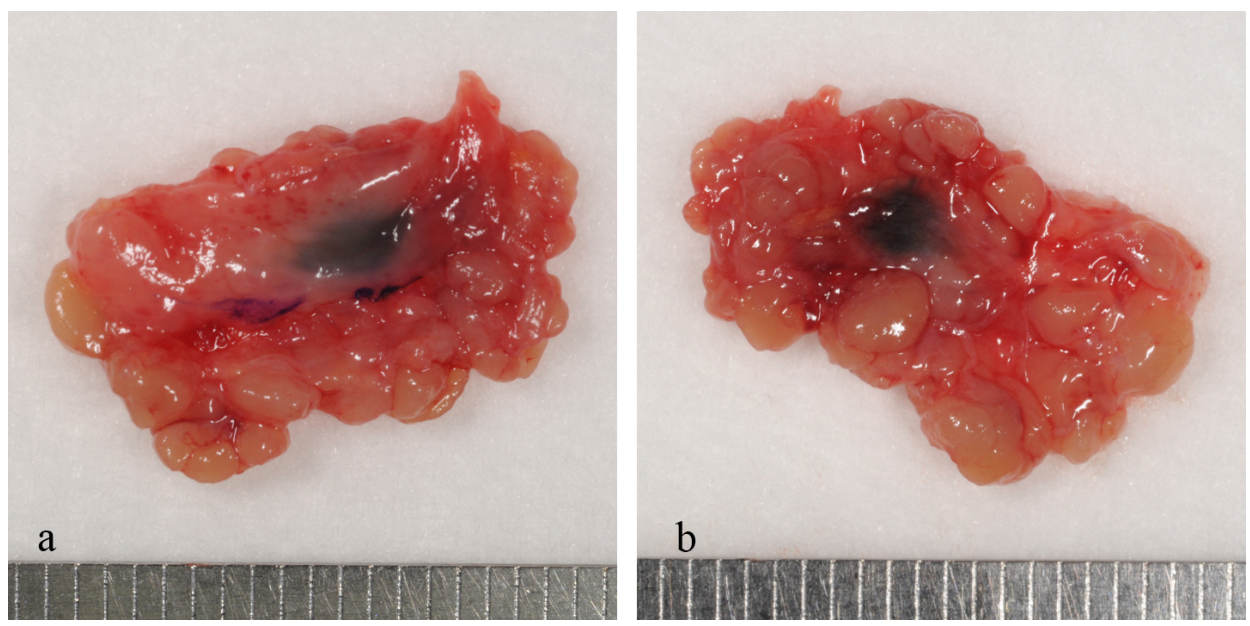

Figure 8. Resected specimen in Case 2: (a) The depth of incision was made to the lipid layer and (b) a dark color was observed on the bottom of the resection stump.
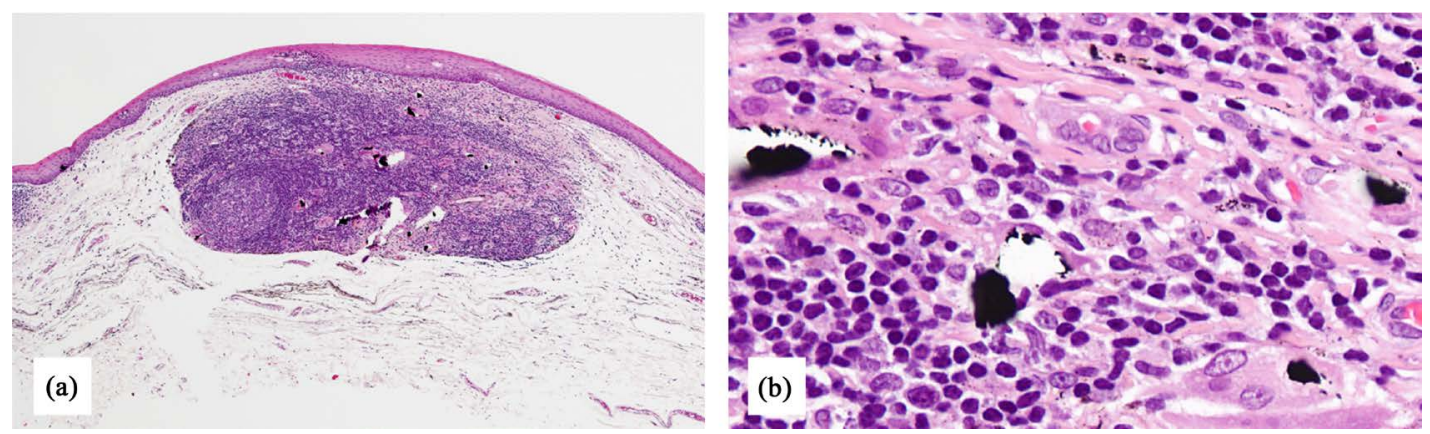

Figure 9. Histopathological images (H \& E stain) in Case 2: Formation of lymphoid tissues underneath the mucosal epithelium and blackish-brown foreign substances were sporadically observed, as well as pigmented dark granules in the collagen fibril (original magnification (a): $\times 2$, (b): $\times 100$ ).

soidal shaped and approximately $20 \mu \mathrm{m}$ in size, with foreign-body type giant cells. Based on these findings, a definite diagnosis of exogenous pigmentation was made.

The metal components were shown by EPMA to be gold (7.4\%), silver (61\%), palladium (9.7\%), copper $(10.8 \%)$, and zinc (4.5\%) (Figure 10). From the results of EPMA, the alloy was revealed to be a gold-silver palladium alloy that is frequently used in dental prosthesis.

Two weeks post-operatively, the area had healed normally and the last follow-up with the patient was 2 months after the surgery uneventfully. 

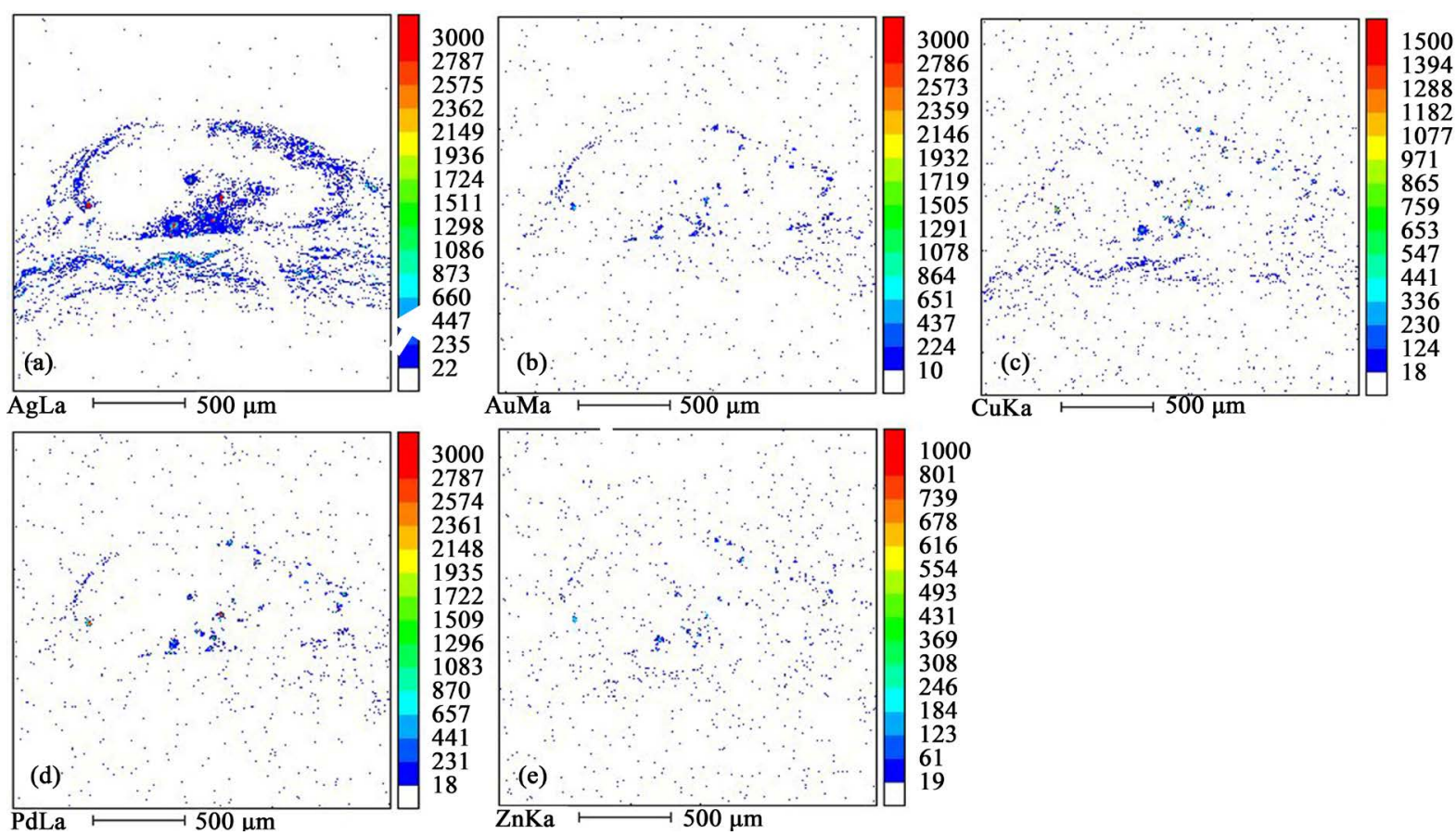

Figure 10. Electron probe microanalysis (EPMA) in Case 2: (a) The metal components were silver 61\%; (b) gold (7.4\%); (c) copper (10.8\%); (d) palladium (9.7\%); and (e) zinc (4.5\%).

\section{Discussion}

Regarding the pigmented lesions of the oral mucosa, exogenous pigmentation due to accidental displacement of dental alloy is a frequently occurring disease in daily clinical practice, and while most cases, except in the cases of aesthetic reasons, remain untreated because they are basically asymptomatic. But, in the case of accurate clinical diagnosis, especially melanoma suspected, it is usually difficult to diagnose upon only inspection and palpation [1]. Differential diagnosis of a pigmented lesion is important to differentiate from malignant melanoma [2]. Adequate image diagnosis based on plain X-ray and CT images is necessary. In the case of exogenous pigmentation due to displacement of dental alloy, aberrant alloy can be normally confirmed as metal-like opacity upon X-ray or CT images [4].

In the present two cases, both cases underwent plain X-ray, ultrasonography, and CT image examinations. In Case 1, while opaque small pieces were observed within the lesion, the opacity was not metal-like but rather cortical bone-like, with a CT value of approximately $2500 \mathrm{HU}$, which is a value similar to that of the mandibular cortical bone. The cause of the low CT value was assessed to be corrosion of the aberrant alloy. On the other hand, in Case 2, no metal-like opacity was observed on CT or panoramic X-ray images because the lesion was small in size. A lower occlusal radiograph may be more effective to detect the aberrant alloy. In both of the cases, no tumor mass formation leading to suspicion of a malignant melanoma was observed on CT images or by ultrasonography, and there were no resorption images of the adjacent mandibular bone. However, in the case of superficial spreading melanoma and melanoma in situ, there is no formation of a tumor mass [5] [6], and the possibility of malignant melanoma could therefore not be entirely ruled out by only imaging findings. Amano et al. reported another case involving an amalgam tattoo on the floor of the mouth, for which malignant melanoma was strongly suspected [2]. In that case, that is similar to Case 2, the blue-black macule, with irregular borders on the floor of mouth did not show opacity by X-rays. Excisional biopsy for differential diagnosis was carried and revealed exogenous pigmentation due to displacement of dental alloy. In Case 1, one pigmented spot was a relatively large bulging lesion, with two more pigmented spots around it, leading to suspicion of satellite lesions of malignant melanoma and making it difficult to rule out the possibility of a malignant melanoma. If malignant melanoma is suspected, it is generally accepted that an excisional biopsy should be carried out due to the possible seeding of malignant cells and high frequency [7]. The policy in our department is also to carry out excisional biopsy of the lesion in cases of suspected and to carry out intra operative rapid diagnosis if necessary. In 
Case 2, while the pigmented spot was relatively small with slight bulging, a definitive clinical diagnosis could not be made preoperatively. The possibility of malignant melanoma could not be completely ruled out in either of the cases, and excisional biopsies were therefore carried out. The incision line was set at $5 \mathrm{~mm}$ from the lesion in both cases, which is considered to be appropriate resection for total removal biopsy without apparent malignant melanoma. Histopathologically, crystal-like foreign substances were detected and were easy to diagnose. The outer periphery of the granular metal foreign substance was fading by metal elution in Case 1 . On the other hand, in Case 2, the foreign substance exhibited a uniform blackish-brown color. Furthermore, similar to findings described in previous reports [1] [3] [4] [8]-[10], blackish-brown fine particles were deposited in the basal membrane of the oral mucosa, the minor salivary duct, peripheral glandular acini, the basal membrane of vessels, and fibroblast cells. In Case 2, there was formation of lymphoid tissues underneath the mucosal epithelium, but an aberrant dental alloy in the mucosa usually causes no body inflammatory reaction or only mild chronic inflammation as previously reported [3] [10]. These difference in tissue responses seem to be related to composition of the aberrant dental alloy because the metal particles were revealed by EPMA to be a silver alloy in Case 1 and a gold-silver palladium alloy in Case 2. EPMA, which is an analytical technique that is used to establish the composition of small areas on specimens and obtain quantitative information, qualitative information, and an element map of a sample, may be effective for identifying the causative dental alloy.

\section{Conclusion}

Although exogenous pigmentation originating from a dental alloy is not rare, differential diagnosis of oral pigmented lesions is sometimes very difficult. In such cases, histopathological examination may be necessary for the diagnosis to exclude melanocytic lesions and EPMA may be effective to identify the causative dental alloy.

\section{References}

[1] Meleti, M., Vescovi, P., Mooi, W.J. and van der Waal, I. (2008) Pigmentated Lesions of the Oral Mucosa and Perioral Tissues: A Flow-Chart for the Diagnosis and Some Recommendations for the Management. Oral Surgery Oral Medicine Oral Pathology Oral Radiology, 105, 606-616. http://dx.doi.org/10.1016/j.tripleo.2007.07.047

[2] Amano, H., Tamura, A., Yasuda, M., Mamanaka, M., Takeuti, Y., et al. (2011) Amalgam Tattoo of the Oral Mucosa Mimics Malignant Melanoma. Journal of Dermatology, 38, 101-103. http://dx.doi.org/10.1111/j.1346-8138.2010.01007.x

[3] Vera-Sirera, B., Risueño-Mata, P., Ricart-Vayá J.M., Baquero Ruíz de la Hermosa, C. and Vera-Sempere, F. (2012) Clinicopathological and immunohistochemical Study of Oral Amalgam Pigmentation. Acta Otorrinolarringologica Espanola, 63, 376-381. http://dx.doi.org/10.1016/j.otorri.2012.02.004

[4] Martin, J.M., Nagore, E., Cremades, A., Botella-Estrada, R., Sanmartin, O., et al. (2005) An Amalgam Tattoo on the Oral Mucosa Related to a Dental Prosthesis. Journal of the European Academy of Dermatology and Venereology, 19, 90-92. http://dx.doi.org/10.1111/j.1468-3083.2004.01071.x

[5] McGovern, V.J., Cochran, A.J., Van der Esch, E.P., Little, J.H. and MacLennan, R. (1986) The Classification of Malignant Melanoma, Its Histological Reporting and Registration: A Revision of the 1972 Sydney Classification. Pathology, 18, 12-21. http://dx.doi.org/10.3109/00313028609090822

[6] Clark Jr., W.H., Ainsworth, A.M., Bernardino, E.A., Yang, C.H., Mihm Jr., C.M., et al. (1975) The Developmental Biology of Primary Human Malignant Melanomas. Seminars in Oncology, 2, 83-103.

[7] Babburi, S., Subramenyam, R.V., Aparna, V. and Sowjanya, P. (2013) Intraoral Malignant Melanoma. Nigerian Medical Journal, 54, 278-281. http://dx.doi.org/10.4103/0300-1652.119667

[8] Aoyagi, H. and Katagiri, M. (2004) Long-Term Effect of Ag-Containing Alloys on Mucous Tissue Present in Biopsy Samples. Dental Materials Journal, 23, 340-347. http://dx.doi.org/10.4012/dmj.23.340

[9] Leit, C.M., Botelho, A.S., Oliveira, J.R., Cardoso, S.V., Loyola, A.M., et al. (2004) Immunolocalization of HLA-DR and Metallothionein on Amalgam Tattoos. Brazilian Dental Journal, 15, 99-103. http://dx.doi.org/10.1590/S0103-64402004000200003

[10] Zhang, X., Gelderblom, H.R., Zierold, K. and Reichart, P.A. (2007) Morphological Findings and Energy Dispersive X-Ray Microanalysis of Oral Amalgam Tattoos. Micron, 38, 543-548. http://dx.doi.org/10.1016/j.micron.2006.07.020 